\title{
Internet of Things in Cloud Environment: Services and Challenges
}

\author{
Karuna Lochab $^{1}$, Dileep KumarYadav ${ }^{1}$, Mayank Singh ${ }^{1}$ and Arun Sharmab ${ }^{2}$ \\ ${ }^{1}$ Krishna Engineering College, Ghaziabad, India \\ karunalochab@gmail.com,dileep252000@gmail.com, \\ mayank.singh@krishnacollege.ac.in, arunsharma2303@gmail.com
}

\begin{abstract}
In this paper evolution of IoT along with its intimacy with cloud is described. Application of IoT in various domains and the services are major research areas and can be enhanced further for more effectiveness and efficiency. Limitations associated with this technique are also addressed and appropriate solutions suggested. The future lies in the 'Internet of Everything'. We have proposed a model which can be used in a wide collection of domains such as by Indian army, military, air-force, navy etc. The main focus of the proposed work is to evolve a combined approach (IoT + Cloud) into a single technology IoTC which will have the benefits of both the technologies and may overcome the shortcomings. It is beneficial in terms of space and time complexity, security, precision and accuracy of result. IoTC can further be applied to services of critically important services.
\end{abstract}

Keywords: IoT, Cloud Computing, Big Data, Data Analytics, Video Surveillance, Biometric, Smart Water

\section{Introduction}

In todays, technological environment, the IoT offers idiosyncratic opportunities for enterprises and organizations to connect hyperactively the people, systems and process. IoT services involves application of IoT technology to the existing real world services such as in big data, cloud computing, video surveillance, medical, mobile applications and many more. IoT is about merging of scattered, relevant and related data but in different formats to seamlessly come together in innovative ways. IoT is the need of hour to modernize the various existing services and develop new services. IoT is an emerging area and is considered to be the future of research as it seamlessly integrates with the host of existing technologies such as cloud computing, big data and many others. IoT is the internetworking of devices (intelligent, connected or smart) embedded with software, electronics, actuators, sensors and network connectivity that renders these devices to exchange and collect data [36-38].

IoT Architecture can be closely related to event-driven model of architecture [39], which was initially formulated as bottom-up( on the basis of operations and processes in real time) but it may further contemplate any auxiliary level. Event in IoT architecture will not essentially stand on syntactic or deterministic model but indeed it will be build on the surroundings or conditions of the occurring of event and will turn out to be a semantic web[40].

The network architecture of IoT demands immense scalability in network design to enable handling of enormous number of devices. With such huge number of devices being amalgamated to internet space, internet(network) layer issue of scalability will be handled by IPv6 protocol[41]. 


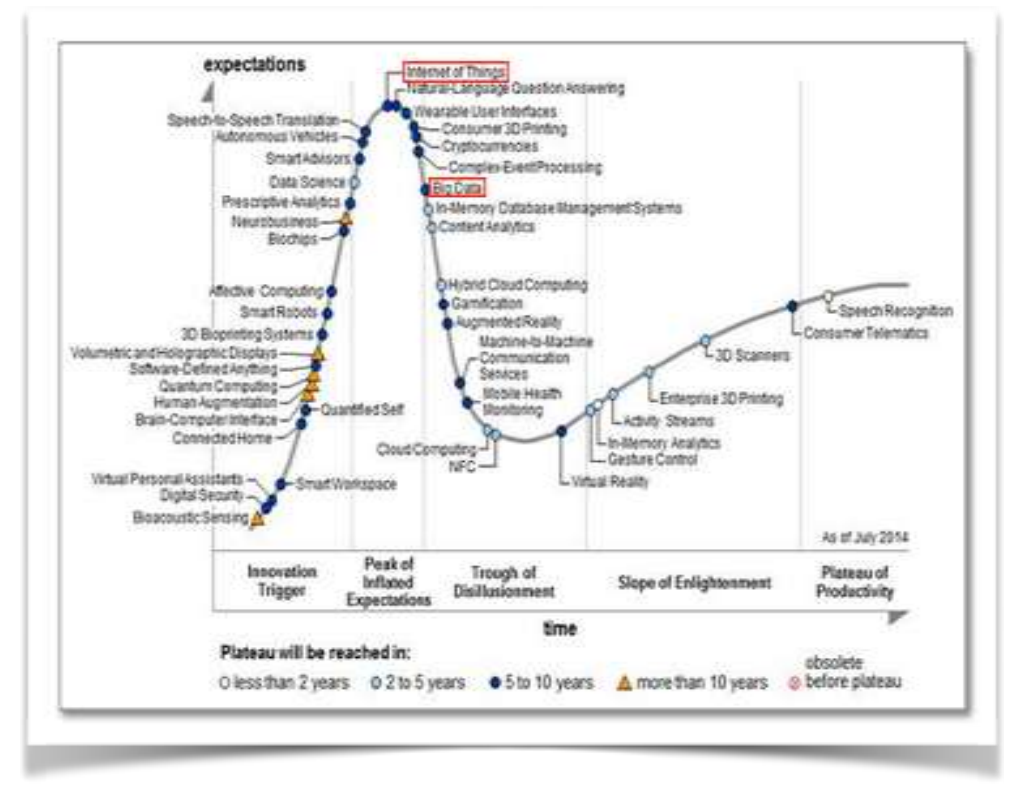

Figure 1. The Hype Cycle [2]

A feasible alternative solution to handle enormous data flow via internet namely 'Fog Computing' can also be used[42].

Its association with cloud makes it more appropriate as the huge amount of data is collected through the intelligent devices which are accommodated in cloud.

However, the IoTC environment is fractioned and requires users and customers to associate with numerous vendors to effectively utilize IoT technology.

\section{Literature Survey}

A novel efficient source discovery mechanism is proposed for SIoT(Social Internet of Things) that works in 3-D cartesian coordinate system. Its takes into account both preference and movement pattern to attain higher degree of search efficiency and minimize system overheads of SIoT[30].

A lot of research was done to support Urban IoTs i.e. Smart City vision aimed at exploiting advanced communication technologies, protocols and architecture for an urban IoT(Padova Smart City project)[31].

Use of IoT devices to obtain automated and human interactive solution to enable high levels of energy savings. A system of inter-connected CPSs(Communicating Power Supply) is developed which enabled communication of control and energy information between the device and other central entities utilizing a custom, cloud based information clearing house[32].

IoT devices are equipped with comprehensive perception, reliable transmission and intelligent processing. In this approach, capability of IoT Applications was explored such as Location Information, Mobile Asset Tracking, Fleet Management, Environment Sensing, Environment Detection, Remote Medical Monitoring, Remote Controlling Appliance Control, Disaster Recovery, Ad-Hoc Network, Secure Communication etc. $[33,34]$.

A set of requirement for IoT Middleware, to address the communication challenges for IoT devices was developed. It addresses issues relating to WSN(wireless sensor network), RFID(radio frequency identification), M2M(machine to machine communication) and SCADA(supervisory control and data acquisition)[35]. 
Latest research is based on security issues of IoT technology. A protocol for secure tracking and tracing of an object was developed. It ensured visibility and traceability of the object along its travel path to support IoT. It is based on RFID system for unique identification of IoT objects globally. The protocol used lightweight cryptographic primitive along with physically unclonable function in tags for ensuring secure object detection and tracking. the protocol was modeled using SPDL(security protocol description language) and simulated using Synther(automated claim verification tool)[36]. A major issue in IoT is space consideration. An accurate and explicit geographic location of a device and also the accurate chronographic dimensions of the thing, is critical[43]. As of now, IoT is being widely used in three core sectors namely home, enterprise and government. The EIoT( enterprise internet of thing ) is the largest among three[44]. There is a host of technologies available to enable IoT, by providing a network that allows for communication between intelligent things of IoT installation. Several wired networks and/or wireless technologies can be used to fulfill the same[45]. Some examples of short range wireless medium are BLE( Bluetooth low energy), Li-Fi( Light-Fidelity), NFC( Near Field Communication), OR Codes, barcodes, RFID( radio frequency identification), Thread( provides IPV6 addressing), Wi-Fi, Wi-Fi Direct, Z-Wave, Zigbee. Medium range wireless networks includes HaLow, LTE-Advanced. Long range wireless like LPWAN( low power wide area network), VSAT( very small aperture terminal)[46]. Some wired networks such as Ethernet, MoCA( multimedia over coax alliance), PLC( power line communication) [47,48].

Simulation, emulation and modeling of IoT is basically done at the design phase. After simulation, deployment of IoT network is done. Simulation can be done using OPNET, Net Sim, NS2, NS3 network simulators[49].

\section{New Initiative: IOTC (Internet of Things with Cloud)}

In this paper, we have explored the domain of Internet of Things with Cloud. It seamlessly integrates the existing different technologies into IoTC. Apart from strengthening the computing power, it also enables storage of huge amount of data on the cloud and use of related historical data already present to conclude to the result as demanded by the applications.

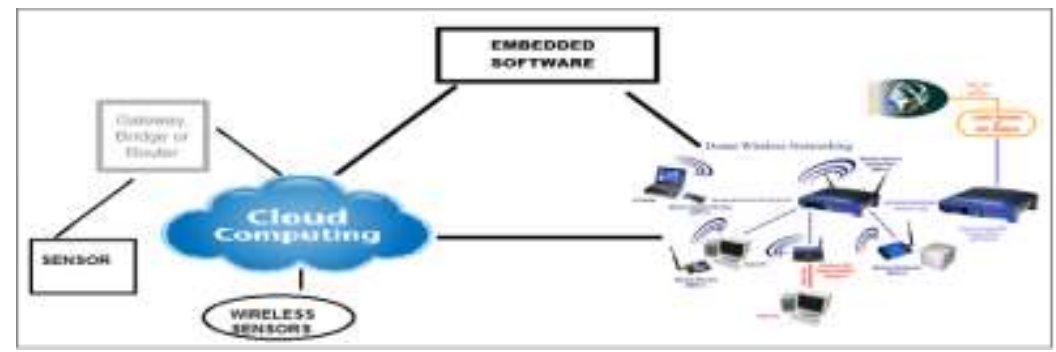

Figure. 2 Working Architecture of IoT in Cloud Environment loTC and Big Data

Big Data is characterized by '4V's' namely volume, variety, veracity and velocity. IoT technology and Big Data are very intimately connected to each other. As numerous IoT devices generates unimaginable amount of complex, multidimensional and heterogeneous data, traditional SQL queried RDBMS( Relational Database Management System) is unsuitable to process such unstructured data. Some emerging technologies such as 'Hadoop', 'No Sql' databases and various business intelligence platforms will be required to adhere to the challenges imposed by such 'target-rich' actionable data that is easily accessible, has a large footprint, available in real time and can bring about meaningful change[1]. 
The research shows the interrelation of internet of things, internet of signs and big data and its effect on the context and on the information drawn from context[29].

\section{IoTC and Data Mining}

The enormous amount of data generated by the IoT devices in various domains such as e-commerce, retail, banking, telecommunications, health care, city governance, flight, satellite etc. is gathered in the cloud. Various Data Mining algorithms can then be applied on such data to extract and analyze invisible information from data, knowledge discovery in databases, data or pattern analysis, data archeology, data dredging and information harvesting[1,3].

Now a days, IoT based Cloud Mining is highly energetic research area and has open problems for researchers.

\section{IoTC in Video Surveillance}

IoTC provides strength to the video surveillance system. This system can be applied to color or thermal imaging to observe, detect, track and recognize the object. It also detects the behavioral activities at geographical borders, sea-port, underwater, air flight, traffic, transportation, robotics, restricted zones, decomposing buildings and monuments. It can also detect and track the movement of crowd and army troops etc. along with their direction of movement[36,37,38].

\section{IoTC in Biometric}

With the advancement and use of IoT in Cloud, Biometric system can be monitored trough IoTC technology. Such systems are observed and visualized by any of the authorized personnel or security persons at the global level. This system can be utilized to detect, track and recognize the pattern of finger, thumb, palm, eye-retina or face etc. Such systems can act as a powerful tool in applications such as attendance monitoring and store the data at cloud[39,40].

\section{IoTC and Data Analytics}

IoT technology and analytics applications offers automatic mechanisms for trailing data from intelligent devices to warehouse. These devices may be smartphones, wearable technology and in-home devices and the data from them which is stored in warehouse is used to perform analysis and to do targeted promotions and marketing, the so called "connected consumer gear" (the business side of the IoT's futuristic world using Cloud Analytics)[4]. Various devices can be used as IoT devices on cloud environment to analyze the required data.

\section{IoTC for Security Purpose in Education}

IoT technology allows to view and keep track of cameras remotely from any system, tablet or smartphone all at same place. IoT along with video surveillance helps to protect students, faculty and staff. It also observes activities of students and staff members inside campus. Such system may be helpful for the purpose of safety and security. These systems act as a protector inside academic institutions. It also provides surveillance and behavioral activity through CCTV, IP cameras which may be connected through cloud and IoT environment and data may be stored and accessed through cloud from anywhere[6]. 


\section{IoTC in Assisted Living}

The information gathered by the IoT devices is used for detecting and tracking unauthorized access to restricted zones, volatile geographical region to implement perimeter access control[5].

\section{Iotc as a Security Measure For Detecting Liquid Presence}

IoTC together with surveillance technique can be used to detect presence of any liquid in sensitive areas such as data centers, data warehouses and other crucial building grounds to prevent corrosion and breakdowns[6]. IoT enabled devices may be able to sense and detect such pitfalls and store the information on the cloud which might be used in future.

\section{Iotc in Radioactive Geographical Areas}

IoT devices can be installed to collect distributed measurement of radiation levels in reactor star or nuclear power plant circumferential to trigger leakage alerts $[5,8,9]$.

\section{Iotc as Security Service in Explosive and Hazardous Gases}

IoT devices can detect any leakages in industrial surroundings and intimation o gas levels in poisonous environment as inside mines and chemical factories $[9,10]$. It also act as prevention process it protect environment and ultimately human life.

\section{Iotc in Retail Industry}

The cloud oriented IoT in retail is focussed on customer's lifetime value(CLV) and customer's referral value(CRV). IoTC can be employed in cash flow calculations, enhances customer's experience, improves operational efficiency and enabling creation of new channels along with revenue streams[17].

\section{Iotc for Supply Chain Control}

The IoT devices may be helpful in SOA(service oriented architecture) to monitor storage conditions alongside the supply chain and tracking of products for traceability[11].

\section{Iotc To Device Intelligent Shopping Applications}

Association rules are employed on the data gathered by IoT devices to help in providing advices to the customers according to their product consumption habits, preferences. IoTC may be helpful in detection of products with expiry dates or with allergic components [12-14].

\section{IoTC in Smart Product Management}

IoT devices automate restocking processes and control of rotation of items in warehouses and grocery store shelves while cloud provides strength to these systems[16]. It also focuses on the IoT technology stack. The embedded software employed using agile approach, communications, cloud platform and for real time applications [17]. 


\section{Implication of IoT in eHealth}

The combination of Big data, cloud and IoT in healthcare domain is attracting customers and organizations through use of IoT devices. These are employed to build fitness devices, other wearables to promote health and well being of people. Some of the wearables that have been developed using IoT integrated techniques includes 'Empatica Embrace', 'Lumo Lift', 'Respironics and Sleepmapper'. Recently Oracle also developed a digital platform and 'Chrono Smart Stop' to promote healthy living standards in society[18].

To provide security in eHealth services a risk driven adaptive security approach 'ASMM'(Adaptive Security Management Model) has been developed. It focuses on security metrics such as security control,its correctness, effectiveness and efficiency. This approach can be further extended for calculating detailed analysis and specification of security metrics and adaptive decision making algorithm[19].

Health solutions were mainly based on SOA( service oriented architecture ), but with the advent of IoT in eHealth a new interface named 'VIRTUS' is evolved which is being used as a middleware. VIRTUS provides XMPP( an instant messaging protocol) to guarantee a real time, reliable and secure communication channel among IoT devices and other associated heterogeneous devices[18].

Recent research in eHealth includes Bio Patch (an unobtrusive Bio Sensor), iMed Pack (intelligent Packaging), iMed Box (intelligent Medicine Box). The fusion of IoT devices with in-Home healthcare services provides for an enhanced user experience and service efficiency[21,50].

\section{Smart Agriculture using IoTC}

IoT technology offers the ability to sense, measure, collect data, infer and understand ecological indicators, from sensitive and delicate environment and natural resources to rural as well as urban environment. The information is then used to develop a COP (common operating picture). IoT merged with Cloud computing (implemented using 'ANEKA') provides for a host of applications in Smart Grid which includes Smart Agriculture, Smart Farming among others for intelligent environment protection[22,23].

Based on major attributes of IoTC and SOA technology massive data in agriculture and farming helped to construct plant factory and implement automatic control production. It further promotes fast evolution of modernization of agriculture, development of Smart Agriculture efficiently. It effectively resolves the issues concerning farmers, countryside and agriculture[24].

IoTC technology can be applied in various domains of agriculture such as enhancement of wine quality by monitoring moisture in soil and trunk radius in vineyards to regulate the quantity of sugar in grapes and grapevine health. It can also be used in Green houses for controlling micro-climate conditions to amplify the production of vegetables and fruits and their quality enhancement. In Meteorological station network to study weather conditions to forecast drought, precipitation mediums such as rain, snow and wind changes. It is also applied in Compost manufacture using control of temperature and humidity levels in hay, alfaalfa, straw etc. to prevent fungus and other types of microbial contaminants. To study the levels of Toxic gases in farms and tracking, identification of animals[5].

\section{Evolution of Smart Water using IoTC}

The regulation of water quality using IoTC technique has vital application in rural as well as urban ecology. It can be used for monitoring of Potable water to calculate the quality of tap water in metropolitan cities. For detection of chemical leakage in rivers by monitoring wastes in rivers and other water bodies.In remote measurement of swimming 
pool by remotely controlling the pool conditions and also to monitor the pollution level in the sea. One of the profound use of IoT is to check and regulate water leakages by detecting presence of liquid outside tanks and pressure measurement and its variation among pipes. It can be also used to monitor water level variations in dams, rivers and reservoirs[5].

A smart water management model 'OPCUA' (object linking and embedding for process control unified architecture) which is a platform independent model was proposed. Here, IoT technology and decision support system provided for efficient water management such as water cost savings and conservation[26].

A novice method based on ICT (information and communication technology) and IoT to suppress the water hummer by precisely controlling of valves[27]. The above method was then evolved to make specific merchant equipment interoperable and easily manageable in this domain homogeneously[28,29].

\section{The Key Benefits of Proposed IoTc Approach}

1. To access, contact and control devices at remote areas.

2. To handle patients and provide prescription to them remotely.

3. Store enormous data on cloud for future use.

4. Saves time and manpower.

5. Saves energy and cost.

6. Get relief from hurdles of managing such huge amount of data.

7. Use of high complexity calculation and availability of sufficient computing space to get precise results.

8. Provides better ways to connect to applications using wide range of platforms.

9. Apart from use of current data, historical data can also be used for arriving at precise results as required by many rigid real time applications.

10. A wide range of security algorithms can be applied to provide security to the information uploaded on the cloud by IoT devices and retrieved information from the cloud by various applications.

\section{Limitations of IoTC}

In order to retrieve optimal results and proper functioning of the IoTC network, naïve software have to be developed to work with hardware components used in intelligent devices in IoT. Further, specific technology platforms are to be developed which will provide such kind of functionality to IoTC and add more intelligence to the existing network. The traditional algorithms used in cloud computing have to be modified and enhanced in order to make them compatible with the IoTC environment. Also, the association rules applied for mining the huge data collected by intelligent things, the security protocols applied in the IoTC network have to be adapted according to the changes in the working scenario.

\section{Conclusion and Future Work}

The existing IoT technology and Cloud Computing are very intimately connected to each other. These technologies are seamlessly combined to give way to a new technology IoTC which provides for a better security and accuracy of results derived by applications. It further reduces the overhead of managing billions and trillions of data collected from IoT devices for computation. IoTC can be applied to various domains such as healthcare, data mining, analytics, agriculture etc. Its can also be applied to applications which require high security of information as in army, military and other services of significant importance. Involvement of IoTC in DIP(Digital Image Processing) and Computer Vision can also be explored. 
IoTC can be combined with various bio-medical science domains such as cognitive science to give rise to Cognitive IoTC( CIoTC) which will be a combination of traditional IoTC and machine learning and intelligence, natural language processing, contextual information and industry specific models.

\section{References}

[1] Y. Chen, A. Han, C. Zhang, "Research On Data Mining Model In The Internet Of Things", International Conference on Automation, Mechanical Control and Computational Engineering, page 156-160, Oct. 2015.

[2] http://www.zdnet.com/article/the-internet-of-things-and-big-data-unlocking-the-power/

[3] F. Chen, P. Deng, J.Wan, D.Zhang, A. V. Vasilakos, X. Rong, "Data Mining for the Internet of Things: Literature Review and Challenges", International Journal of Distributed Sensor Networks, vol. 11, no. 8431047, page 1-14, Aug. 2015.

[4] http://searchbusinessanalytics.techtarget.com/essentialguide/IoT-analytics-guide-UnderstandingInternet-of-Things-data

[5] http://www.libelium.com/resources/top_50_iot_sensor_applications_ranking/

[6] T.P. Chen, H. Haussecker, A. Bovyrin, R. Belenov, K. Rodyushkin, A. Kuranoc, V. Eruhimov, “ Computer Vision Workload Analysis: Case Study of Video Surveillance System”, Intel Technology Journal, Vol. 9 Issue 2, p109-118, May. 2005.

[7] http://www.ifsecglobal.com/smart-cctv-and-the-internet-of-things-2016-trends-and-predications/

[8] http://www.innovationessence.com/internet-applications-smarter/

[9] http://blog.cubeacon.com/4-internet-of-things-implementation-for-security-and-emergency.html

[10] Vanimireddy, D.A. Kumari, "Detection of Explosives Using Wireless Sensor Networks", International Journal of Engineering Trends and Technologies, vol. 3, page 277-280, 2012.

[11] L.D. Xu, "Information Architecture For Supply Chain Quality Management", International Journal of Production Research, vol.1 Issue 1, page 183-198, Nov. 2010.

[12] L. Coetzee, J. Eksteen, "The Internet of Things- Promise for the Future? An Introduction", IST Africa Conference 2011, page 1-9, Aug. 2011.

[13] F. Tao, Y. Zuo, L.D. Xu, L. Zhang, "IoT- Based Intelligent Perception and Access of Manufacturing Resource Toward Cloud Manufacturing”, IEEE Transactions on Industrial Informatics, vol. 10, Issue 2, page 1547-1557, May. 2014.

[14] M. Yun, B. Yuxin, "Research on the Architecture and Key Technology of Internet of Things applied on Smart Grid",International Conference on Advances in Energy Engineering, no. 11499733, Jun. 2010.

[15] http://www.iot-now.com/2016/01/29/41675-iot-in-retail-transformation-of-the-shopping-experience/

[16] http://techproductmanagement.com/iot-primer/

[17] http://www.swaransoft.com/retail.html

[18] M. Bazzani, D. Conzon, A. Scalera, M.A. Spirito, C.I. Trainito, "Enabling the IoT Paradigm in E-health Solutions through the VIRTUS Middleware", IEEE 11th International Conference on Trust, Security and Privacy in Computing and Communications, pages 1954-1959, Nov. 2012.

[19] R.M. Savola, H. Abie, M. Sihvonen, "Towards Metrics-Driven Adaptive Security Management In EHealth IoT Applications", SETTIT 2012, page 125-137, May. 2012.

[20] G. Yang, X. Li, M. Mantysalo, X. Zhou, Z. Pang, L.D. Xu, S.K. Walter, Q. Chen, L. Zheng, "A HealthIoT Platform Based on the Integration of Intelligent Packaging, Unobtrusive Bio-Sensor and Intelligent Medicine Box", IEEE Conference on Industrial Informatics,vol.10, Issue-4, page 2180-2191, May. 2014.

[21] J. Gubbi, R. Buyya, S. Marusic, M. Palaniswami, "Internet of Things (IoT): A Vision, Architectural Elements, and Future Directions", Future Generation Computer Systems, page 965-984, vol. 28, issue 7, Jul. 2012.

[22] L. Li, H. Xiaoguang, C. Ke, H. Ketai, "The applications of WiFi-based Wireless Sensor Network in Internet of Things and Smart Grid", $6^{\text {th }}$ IEEE Conference on ICIEA, page 123-131, Sep. 2014.

[23] F. TongKe, "Smart Agriculture Based on Cloud Computing and IoT", Journal of Convergence Information Technology, Volume 8, Number 2, Jan 2013 doi : 10.4156/jcit.vol8.issue2.26

[24] https://www.thingworx.com/ecosystem/markets/smart-connected-systems/smart-agriculture/

[25] T. Robles, R. Alcarria, D. Martin, M. Navarro, R. Calero, S. Iglesias, M. Lopez, "An IoT based Reference Architecture for Smart Water Management Processes", Journal of Wireless Mobile Networks, Ubiquitous Computing and Dependable Applications, vol. 6, number 1,Jul. 2014, pages 4-23.

[26] K. Takahiro, S. Naofumi, T. Masatake, T. Shin, M. Sumio, I.Stoianov, "Smart Water Management Technology with Intelligent Sensing and ICT for the Integrated Water Systems", NEC Technical Journal, Vol.9, No.1, Special Issue on Solutions for Society - Creating a Safer and More Secure Society, Jan. 2013, pages 103-107.

[27] T. Robles, R. Alcarria, D. Martin, M. Navarro, R. Calero, S. Iglesias, M. Lopez, "An Internet of Things based model for Smart Water Management", 28th International Conference on Advanced Information Networking and Applications Workshops, May. 2014, pages 821-826, doi 10.1109/WAINA.2014.129. 
[28] D. E. O'Leary, “'BIG DATA', THE 'INTERNET OF THINGS' AND THE 'INTERNET OF SIGNS'”, Intelligent Systems In Accounting, Finance and Management, pages 53-65, Nov. 2013.

[29] L. Guojie, C. Xueqi, "Research Status and Scientific Thinking of Big Data", CNKI, pages 212-219, Jul. 2012.

[30] Zanella, N. Bui, A. Castellani, L. Vangelista, M. Zorzi, “IoT for Smart Cities”, IEEE Internet of Things Journal, vol.1, Issue 1, pages 22-32, Feb. 2014

[31] S. Lanzisera, A.R. Weber, A. Liao, D. Pankaj, A.K. Mier, "Communicating Power Supplies: Bringing the Internet to the Ubiquitous Energy Gateways of Electronic Devices", IEEE Internet of Things Journal, vol.1, Issue 2, pages 152-160, Mar. 2014.

[32] S. Chen, H. Xu, D. Liu, B. Hu, H. Wang, "A Vision of IoT: Applications, Challenges and Opportunities with China Perspective", IEEE Internet of Things Journal, vol.1, issue 4, pages 349-359, Jul. 2014.

[33] J.A. Stankovic, "Research Direction for the IoT", IEEE Internet of Things Journal, vol.1, issue 1, pages 3-9, Feb. 2014.

[34] M.A. Razzaque, M.M. Jevric, A. Palade, S. Clarke, "Middleware for Internet of Things: A Survey", IEEE Internet of Things Journal, vol. 3, issue 1, pages 170-195, Feb. 2016.

[35] B.R. Ray, M.U. Chowdhury, J.A. Abawajy, "Secure Object Tracking Protocol for the Internet of Things", IEEE Internet of Things Journal, vol. 3, issue 4, pages 544-553, Aug. 2016.

[36] Brown, Eric (13 September 2016). "Who Needs the Internet of Things?". Linux.com.

[37] Brown, Eric (20 September 2016). "21 Open Source Projects for IoT". Linux.com.

[38] "Internet of Things Global Standards Initiative". ITU. Retrieved 26 June 2015.

[39] Gautier, Philippe, "RFID et acquisition de données évènementielles : retours d'expérience chez Bénédicta", French Journal of Management Information Systems, pages 94-96, 2016.

[40] Fayon, David (17 April 2010). "3 questions to Philippe Gautier". i-o-t.org.

[41] "Gartner Says 6.4 Billion Connected "Things" Will Be in Use in 2016, Up 30 Percent From 2015". .

[42] A. Pal, "Making the Hype a Reality", IEEE Computer Society, Jun. 2015.

[43] "OGC Sensor Things API standard specification". OGC. Feb. 2016.

[44] "The Enterprise Internet of Things Market". Business Insider. 25 February 2015. Retrieved 26 June 2015.

[45] Want, Roy; Bill N. Schilit, Scott Jenson (2015). "Enabling the Internet of Things". 1. Sponsored by IEEE Computer Society. IEEE. pp. 28-35.

[46] Al-Fuqaha, A.; Guizani, M.; Mohammadi, M.; Aledhari, M.; Ayyash, M. (2015-01-01). "Internet of Things: A Survey on Enabling Technologies, Protocols, and Applications". IEEE Communications Surveys Tutorials. 17 (4):23472376. doi:10.1109/COMST.2015.2444095. ISSN 1553-877X.

[47] "The Internet of Things: a jumbled mess or a jumbled mess?". The Register. June 2016.

[48] "Can we talk? Internet of Things vendors face a communications 'mess'". Computerworld. June 2016.

[49] P. Sharma, K. Singhal, Neha, M. Dixit, R. Singh, S. Gill, "A REVIEW ON IOT-HAS VIA WIFI MODULE", International Journal of Scientific Research and Management Studies (IJSRMS), Vol. 3, Issue 7, pages 264-270, Jan.2016.

[50] K. Murthy, B. Heeramani, B. Nagaraj, "Research of Smart Business Marketing Techniques by Internet of Things for Global Trading in 2017", International Journal of Science Technology and Management, vol. 5, issue. 12, pages 659-669, Dec. 2016. 
International Journal of Database Theory and Application Vol.10, No.5 (2017) 